\title{
Investigation on Laser-annealing and Subsequent Laser-nanotexturing of Amorphous Silicon (a-Si) Films for Photovoltaic Application
}

\author{
I.A. Palani ${ }^{* 1}$, N.J. Vasa ${ }^{{ }^{2}}$, M. Singaperumal ${ }^{* 1}$, T. Okada ${ }^{* 3}$ \\ ${ }^{* 1}$ Department of Mechanical Engineering, Indian Institute of Technology Madras, \\ Chennai-600036, India \\ E-mail:palaniia@gmail.com,msingam@iitm.ac.in \\ ${ }^{* 2}$ Department of Engineering Design, Indian Institute of Technology Madras, \\ Chennai-600036, India \\ E-mail: njvasa@iitm.ac.in \\ ${ }^{* 3}$ Graduate School of Information Science and Electrical Engineering (ISEE), \\ Kyushu University, Fukuoka 319-0095, Japan \\ Email: okada@ees.kyushu-u.ac.jp
}

\begin{abstract}
Development of textured crystalline silicon from amorphous silicon film using a pulsed $\mathrm{Nd}^{3+}$ :YAG laser is reported. The technique involved is laser annealing and subsequent laser nano texturing by over lapping the laser spot by $50 \%$ and $90 \%$ of the diameter. Experimental investigations were performed using third $(355 \mathrm{~nm})$, second $(532 \mathrm{~nm})$ and fundamental harmonics $(1064 \mathrm{~nm})$ of the pulsed $\mathrm{Nd}^{3+}$ :YAG laser. To investigate the texturization AFM measurement was performed. Absorbance and photoconductivity measurements were performed to analyze the photovoltaic properties of thin films. The process was theoretically analyzed using a 3-D heat transformation model in FEMLAB software. $355 \mathrm{~nm}$ laser assisted nano-texturing with 90\% overlap showed an improvement in crystalline property and improved textured surface with a higher absorbance as compared to that of $50 \%$ overlap.

DOI: $10.2961 / \mathrm{j} l \mathrm{mn} .2010 .02 .0010$
\end{abstract}

Keywords: laser annealing, laser nano-texturing, $\mathrm{Nd}^{3+}$ : YAG, amorphous silicon (a-Si), polycrystalline silicon, Photovoltaic application

\section{Introduction}

Polycrystalline-silicon (poly-Si) thin film has been of great research interest for applications as active layers in thin film transistors and solar cells. In substitute of polycrystalline silicon, low-cost amorphous-silicon films can be considered for photovoltaic applications. a-Si films possess a larger band gap $(1.7 \mathrm{eV})$ than crystalline silicon. Hence, they absorb the visible part of the solar spectrum more effectively than the infrared portion of the spectrum. But the efficiency is limited due to the absence of crystalline structure $[1,2]$.

In addition to the above factors, the efficiency of a solar cell is determined by its ability to gather light. A suitable textured surface can play a significant role in improving the amount of incident light absorption through multiplereflections. This requires creation of a roughened surface so that the incident light can be absorbed by the solar cell through multiple reflections. Even though there are different texturization methods, such as acidic texturing, ion etching etc., these techniques are generally used for monocrystalline silicon [3,4]. Laser assisted annealing and subsequent texturing of a-Si films, proposed in this work is also expected to improve the efficiency of light absorption.

Laser assisted annealing of a-Si films has been considered for various applications, like thin-film-transistor display [5]. When short, high-intensity laser pulses are used to heat amorphous silicon thin films around the melting point, crystallized Si starts to nucleate and grow as it cools. Laser crystallization of a-Si has been a subject of intense research for a considerable time [6,7]. The most widely used method for laser crystallization of a-Si is based on excimer lasers. The principal advantage of excimer lasers is the strong absorption of UV light in silicon. Most of the laser energy is deposited close to the surface of the thin film and the thermal strain on the substrate is much lower than in the case of lasers with longer wavelength, resulting in an efficient crystallization. Excimer lasers, such as $\mathrm{XeCl}$ (308 $\mathrm{nm}$ ) and $\mathrm{KrF}(248 \mathrm{~nm})$, are widely used for annealing of a-Si films $[8,9]$. However, they have limitations, such as (i) high maintenance cost, (ii) high operational cost, and (iii) environmental problems. Further, for photovoltaic applications, laser-assisted treatment of few hundreds of nm thick a-Si film is necessary.

In the present study, an attempt is made to crystallize and subsequently form a textured surface using a solid-state $\mathrm{Nd}^{3+}$ :YAG laser (Nd:YAG laser) at three wavelengths (355, 532 and $1064 \mathrm{~nm}$ ) on a-Si films by 50 and $90 \%$ overlapping of the diameter of the laser spot while laser annealing of the film. Treated samples of a-Si films are characterized for extent of crystallization, surface morphology, electrical and optical properties. Laser annealing with spot overlap is simulated using FEMLAB software package. 


\section{Experimental Investigation}

Laser annealing and subsequent laser nano-texturing were performed using a solid-state pulsed Nd:YAG laser (Quanta Ray GCR 100 series). Experiments were conducted at three wavelengths $(355,532$ and $1064 \mathrm{~nm})$, with a pulse repetition rate of $10 \mathrm{~Hz}$ and a pulse width of $9 \mathrm{~ns}$. aSi thin films $(400 \mathrm{~nm})$ were coated on two substrates: alkali free glass $(0.5 \mathrm{~mm}$ thick $)$ and n-type c-Si $(0.5 \mathrm{~mm}$ thick). The samples were cut into $15 \mathrm{~mm} \times 15 \mathrm{~mm}$ square pieces and chemically cleaned and mounted on an X-Y stage. The laser beam was focused on a-Si thin film samples using a convex lens of focal length $300 \mathrm{~mm}$ to obtain the spot size of $2 \mathrm{~mm}$. The laser pulse was controlled for single pulse treatment. The laser treatment was done at atmospheric condition.

In the case of laser annealing and subsequent laser nanotexturing, the laser pulse is first made incident on the surface of the a-Si. After the phase transformation is completed during the pulse to pulse duration of $3 \mathrm{~s}$ the next laser spot is made to overlap with the previous irradiation spot to a certain predetermined extent. In the study, to induce texturization two trials were conducted in which the samples were annealed with each subsequent laser beam overlapped at $50 \%$ and $90 \%$ in terms of the diameter of the laser spot. Fig. 1 shows pictorially the overlap of the consecutive laser beams and three regions, namely irradiated regions corresponding to the preceding and succeeding laser pulses; and the overlapping interface region. To maintain uniformity, the overlapping was done along both the $\mathrm{X}$ and $\mathrm{Y}$ axes. To investigate the influence of the laser fluence on the annealing and the subsequent texturing, a-Si films were treated with different fluence values by altering the laser beam energy.
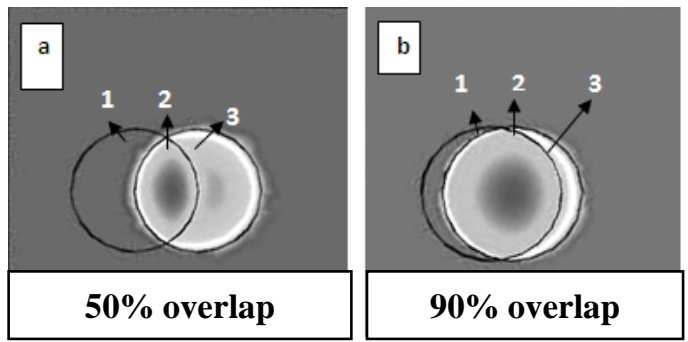

1. Laser crystallized silicon (first spot),

2. Interface region where laser spots overlap, 3. Laser irradiated (second spot)

Fig. 1 Regions of laser spot coverage during annealing and nano-texturing.

The surface morphology of the annealed samples were analyzed using a scanning electron microscope (SEM) (Keyence VE-7800). The extent of crystallization of the aSi films were characterized by using the Raman spectroscopy (Jusco 5000 series) technique. The texturization on the samples were analyzed though AFM (Keyence VN8000). Electrical resistance of samples was measured by using a precision multi-meter (Keithley 2000 series) and the optical properties of the laser textured samples were analyzed using an UV spectrometer (Shimadzu UV-160A). Aluminum coplanar electrodes were deposited on the film and photoconductivity was measured at room temperature using an I-V measurement system (HP4145B) and a solar simulator (WXS-155S-L2, WACOM) with a Xenon lamp as the illumination source.

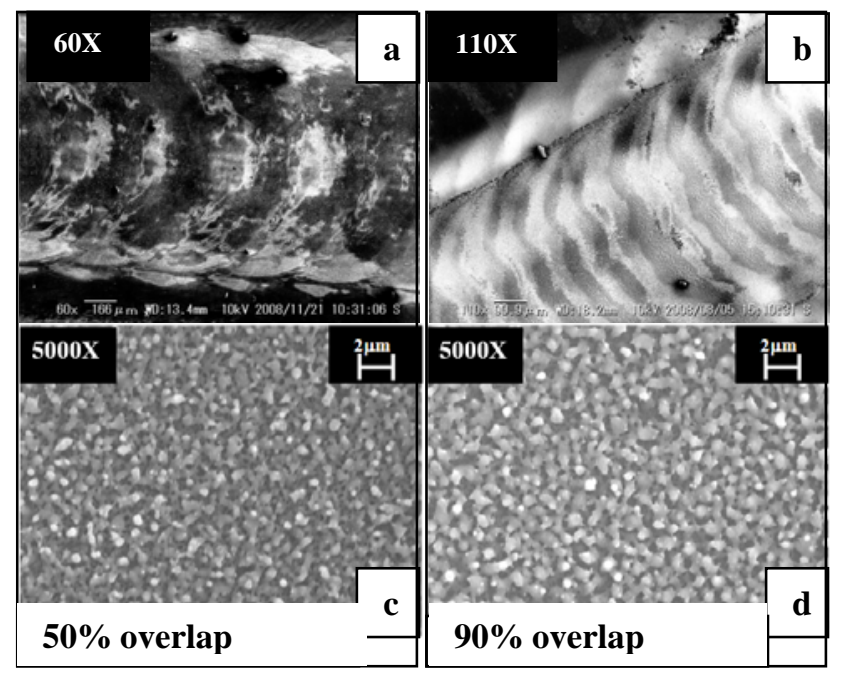

Fig. 2 SEM micrograph of laser treated a-Si/c-Si. Laser wavelength $=355 \mathrm{~nm}$ and laser fluence $=470$ $\mathrm{mJ} / \mathrm{cm}^{2}$.

\section{Results and Discussion}

\subsection{Laser crystallization of a-Si at three different \\ Wavelengths}

Fig. 2(a), (c) and (b), (d) show SEM micrographs with different magnification at $50 \%$ and $90 \%$ beam overlapping, respectively. The treatment was performed at a laser wavelength of $355 \mathrm{~nm}$ and with a laser fluence of $470 \mathrm{~mJ} / \mathrm{cm}^{2}$. As seen from Fig. 2, the surface morphologies of film produced with $50 \%$ and $90 \%$ overlap are different.
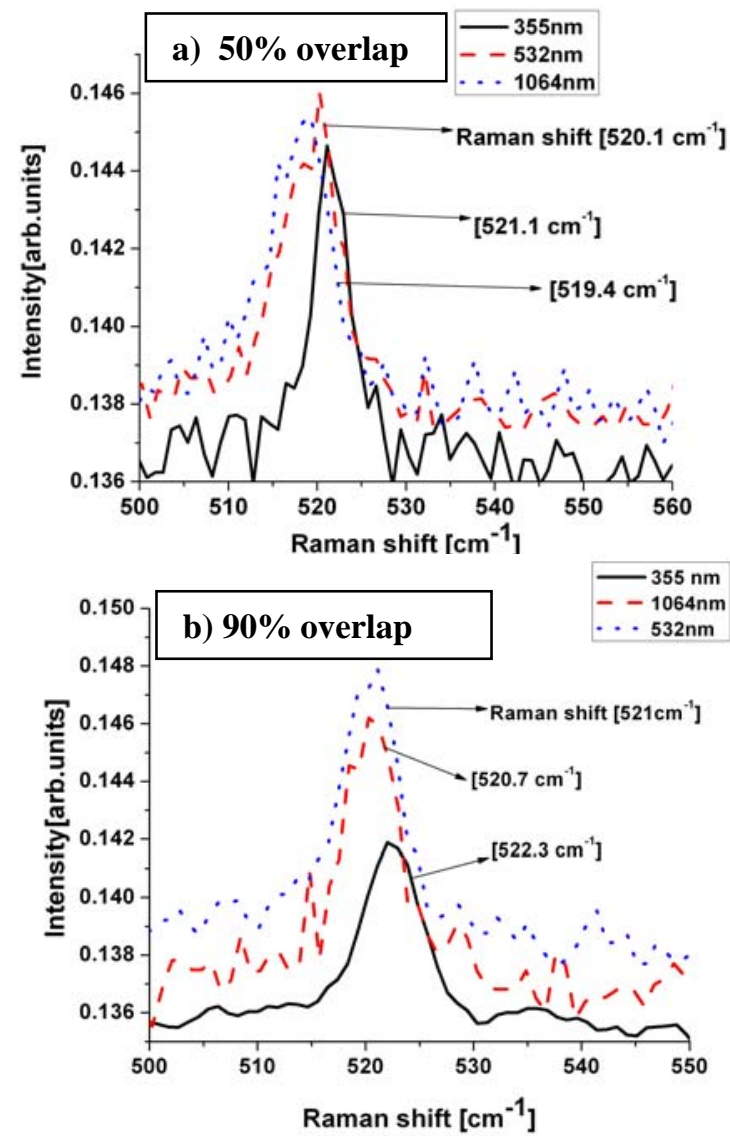

Fig. 3 Raman spectroscopy of laser treated a-Si/c-Si. 
Fig. 3(a) and (b) show Raman spectra of a-Si film samples treated at $50 \%$ and $90 \%$ overlap at different wavelengths. Without laser treatment, a broadband spectrum around $475 \mathrm{~cm}^{-1}$ was observed representing amorphous nature of the film. On laser annealing, typically Raman peak around $520 \mathrm{~cm}^{-1}$ is observed for all wavelength. a-Si film samples were treated at the third harmonics $(355 \mathrm{~nm})$, the second harmonics $(532 \mathrm{~nm})$ and the fundamental wavelength (1064 nm), with typical fluence values of 480, 500 and $1190 \mathrm{~mJ} / \mathrm{cm}^{2}$, respectively. The Raman scattering peak values with $50 \%$ and $90 \%$ overlap are comparable and no significant difference is observed.
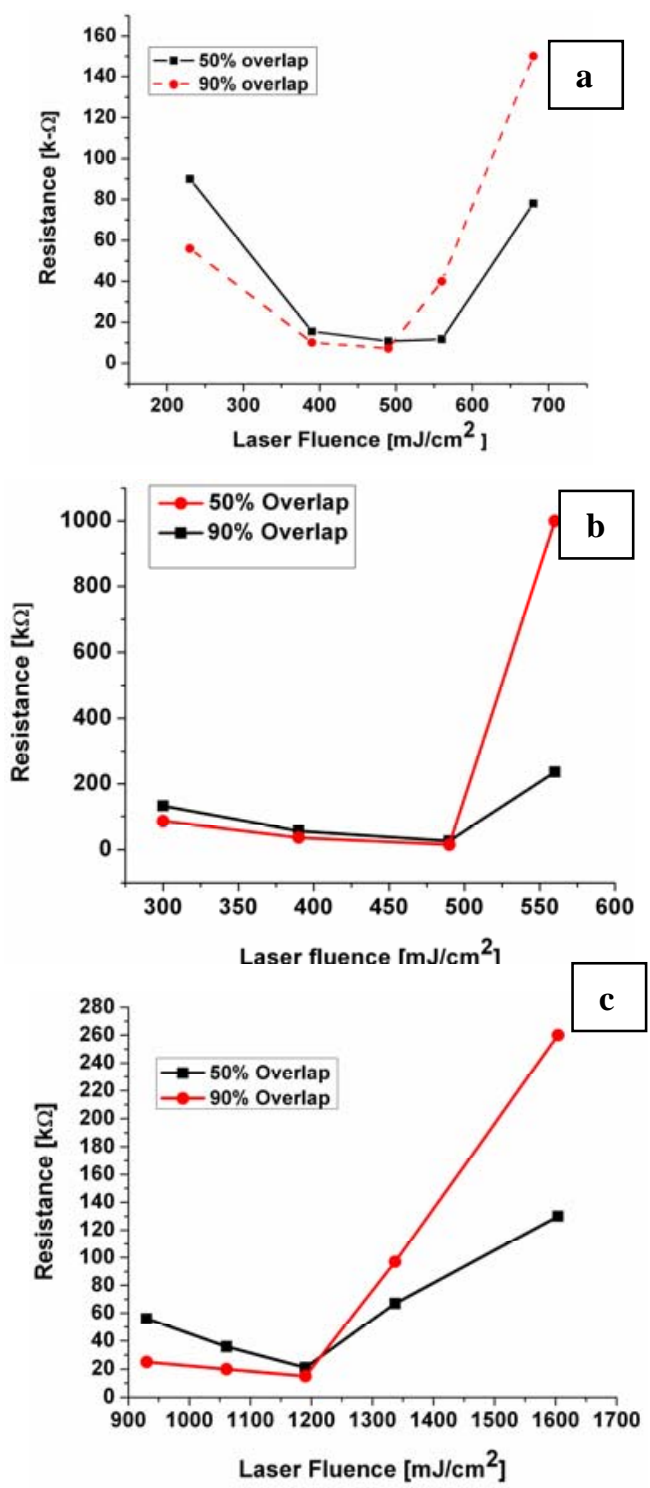

Fig. 4 Resistance measurement of samples treated with a wavelength a) $355 \mathrm{~nm}$ b) $532 \mathrm{~nm}$ c) $1064 \mathrm{~nm}$.

Fig. 4(a), (b) and (c) show variation in electrical resistance with respect to laser fluence values for a-Si/c-Si samples when treated with different laser wavelengths, and at $50 \%$ and $90 \%$ overlap of the laser spot. The resistance of an a-S/c-Si sample without any laser treatment was measured to be $150 \mathrm{M} \Omega$. As shown in Fig. 4, a drop in resistance is observed with increase in the laser fluence values at all wavelengths. When samples were treated with the laser fluence higher than the ablation threshold, the resis- tance values increase rapidly. This might be due to the ablation effect. Due to the influence of an uncontrolled melting, an amorphous region in the substrate was resulted. As shown in Fig. 4, there exists a process-window in which the resistance values of the laser treated films are lower. With $90 \%$ overlap, the resistance drop is slightly higher than that of the $50 \%$ overlap. Samples treated with the third harmonics $(355 \mathrm{~nm})$ show the lowest resistance of $7 \mathrm{k} \Omega$ at $90 \%$ overlap. A slight difference in electrical resistance characteristic was observed when the samples were treated at $50 \%$ or $90 \%$ overlap. Also when the samples were treated with $90 \%$ overlap, electrical resistance of the films was a minimum at lower laser fluence as compared to that of the $50 \%$ overlap. This confirms the improvement in electrical characteristic of a-Si films treated with $90 \%$ overlap. No significant variation in the resistance was observed between the samples treated with $355 \mathrm{~nm}$ and $532 \mathrm{~nm}$. However when the samples were treated with $1064 \mathrm{~nm}$, the laser fluence required to achieve minimum resistance values was considerably higher and the minimum resistance reached was around $20 \mathrm{k} \Omega$. This might be due to the low absorbance leading to heating of the substrate to a larger extent resulting in a complex crystallization process and the filmsubstrate interface damage [10].

The samples treated with a wavelength of $355 \mathrm{~nm}$ were also characterized using a Hall-effect measurement setup. The Hall mobility was estimated to be around $897 \mathrm{~cm}^{2} / \mathrm{Vs}$. Similar Hall-effect characteristics were also reported by other researchers with an application of a $\mathrm{KrF}$ excimer laser $(248 \mathrm{~nm})$, and the results obtained are broadly comparable [11].

\subsection{Investigation on textured crystallized silicon}

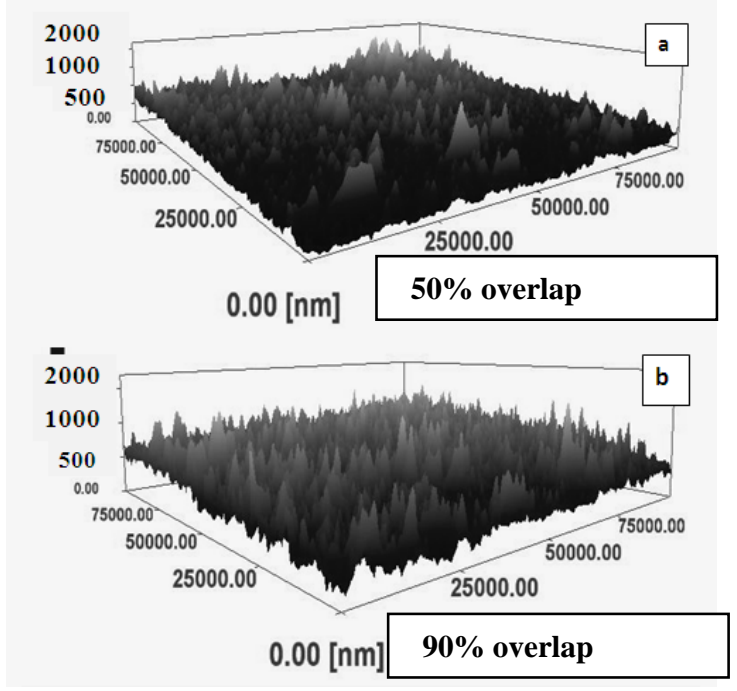

Fig. 5 AFM images of a-Si film samples treated a laser wavelength of $355 \mathrm{~nm}$ and a laser fluence of $470 \mathrm{~mJ} / \mathrm{cm}^{2}$.

Influence on texturization of a-Si film samples during the laser annealing treatment was studied with $355 \mathrm{~nm}$ laser wavelength at a laser fluence of $470 \mathrm{~mJ} / \mathrm{cm}^{2}$. Figures 5 (a) and (b) show AFM images of the laser textured a-Si film samples at $50 \%$ and $90 \%$ overlap, respectively. From Fig. 5, it is seen that a larger amount of surface peaks exists with $90 \%$ overlap than with $50 \%$ overlap. 
Fig. 6(a) shows variation of surface roughness parameter (Rq, which is also termed as root mean square of surface roughness) measurement at $50 \%$ and $90 \%$ overlap with laser fluence for two different substrates. The increase in surface roughness at $90 \%$ overlap might be due to the increase in melt zone as compared to that of $50 \%$ overlap at the interface. Significant variation in $\mathrm{Rq}$ was not observed when the laser fluence was less than the damage threshold value. However, when the laser fluence is higher than the damage threshold fluence, as seen in Fig. 6(a), the value of $\mathrm{Rq}$ is reduced for the a-Si/glass sample and rapidly increased for the a-Si/c-Si sample.

Film samples with $90 \%$ overlap show higher RMS values in the region of crystallization suitable for incident light trapping, and hence when the laser fluence value reaches the ablation threshold, a reduction in RMS value is observed. Figure 6(b) shows the SEM micrograph of the textured a-Si/c-Si taken at an inclination of $25^{\circ}$ showing the textured p-type silicon of around $1 \mu \mathrm{m}$ size on the n-type crystalline substrate
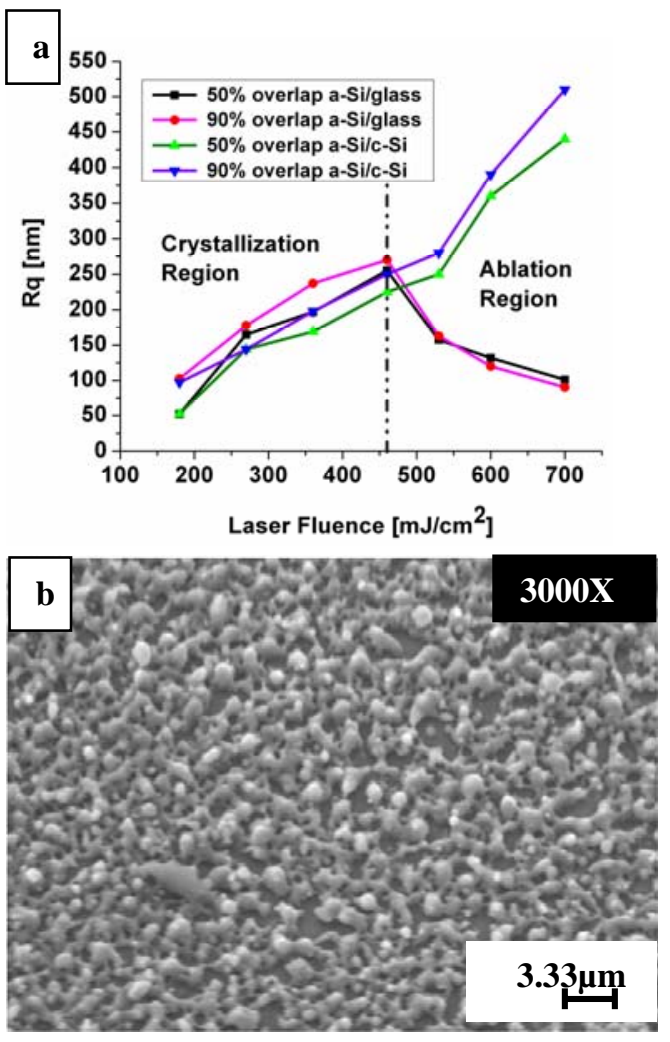

Fig. 6 (a) surface roughness RMS Vs laser fluence at $355 \mathrm{~nm}$, (b) SEM micrograph of laser textured $\mathrm{Si}$.

\subsection{Photoconductivity and optical properties of laser crystallized and nano-textured a-Si}

Photovoltaic properties, such as light absorbance and photoconductivity, of the laser treated and nano-textured a$\mathrm{Si} /$ glass samples were investigated.

Figure 7 shows spectroscopy measurements in terms of absorbance $\left(=-\log _{10}\left(I / I_{0}\right)\right.$, where $I=$ transmitted light intensity, $I_{0}=$ incident light intensity) of the samples at $50 \%$ and $90 \%$ overlap. Based on Fig. 7, at a typical wavelength of $1100 \mathrm{~nm}$, the absorbance for the untreated a-Si/glass sample was around 0.5 .

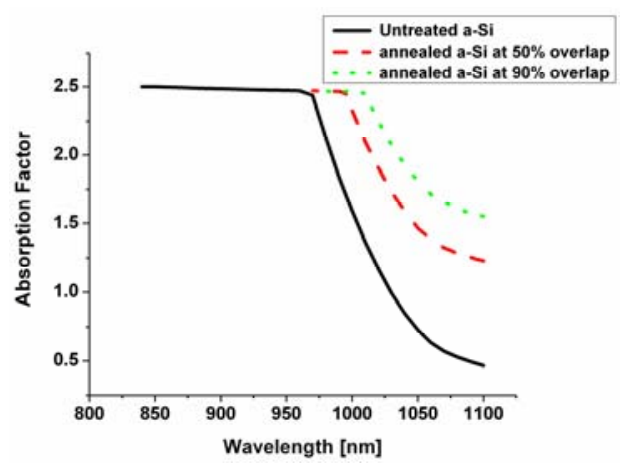

Fig. 7 Absorption spectra of a-Si/glass samples.

On the other hand, the absorption factor value was improved to 1.25 and 1.68 for samples textured with $50 \%$ overlap and $90 \%$ overlap, respectively.
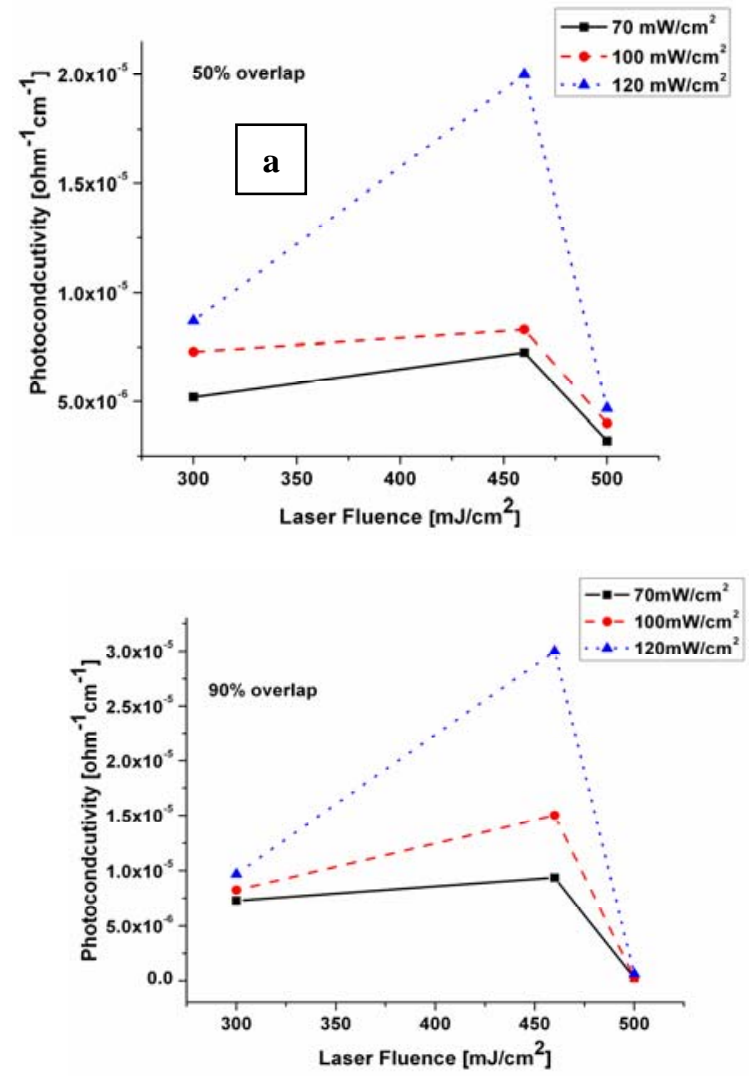

Fig. 8 Photoconductivity measurements.

This measurement clearly indicates that texture formed using laser treatment of a-Si samples with $90 \%$ overlap improved the light absorbance characteristics.

Figures 8(a) and (b) shows the result of photoconductivity measurements of nano-textured, un-doped a-Si/glass samples at 50 and $90 \%$ overlap, respectively. Measurements were performed for a-Si films treated with different laser fluence values and three typical illumination intensities of 70,100 and $120 \mathrm{~mW} / \mathrm{cm}^{2}$. The maximum photoconductivity of $3 \times 10^{-5} \Omega^{-1} \mathrm{~cm}^{-1}$ was observed on a-Si samples treated with $90 \%$ overlap at a fluence of $470 \mathrm{~mJ} / \mathrm{cm}^{2}$. 


\subsection{Modeling}

Laser annealing of a-Si using a single pulse is a combination of two steps: i) rapid melting of a-Si film through absorption of laser radiation resulting in formation of polycrystalline grains from the nucleation sites that are either have been present in the film prior to the exposure (and remain unaffected by it) or formed as the liquid cools down $[12,13]$; ii) formation of surface structure due to the thermo-capillary force where the surface tension and the viscosity play a major role in uplifting the molten silicon. This results in a surface modification on the crystallized silicon surface [14]. The phase transformation during laser annealing takes place in a very short time span of few nanoseconds.

In the case of laser annealing and subsequent laser nano-texturing, the first laser pulse is made incident on the surface of the a-Si. After the phase transformation is completed the second laser spot is made to overlap with the previous irradiated spot to a desired extent. In the modeling, pulse to pulse duration was maintained at $3 \mathrm{~s}$ similar to that of the experimental pulse duration. Due to the variation in properties at the boundaries of the interface, difference in thermal gradient is expected, which may result in variation in texturing of thin film $[15,16]$. Three different regions on the irradiated target are considered when the laser beam overlapping is used: 1) laser crystallized region due to the first pulse, 2) an interface region, 3) a-Si being crystallized due to the second pulse. These regions have different thermal properties as shown in Table 1 [17-19].

Table 1 Thermo-physical properties of silicon

\begin{tabular}{ccc}
\hline $\begin{array}{c}\text { Re- } \\
\text { gion }\end{array}$ & $\boldsymbol{k}[\mathbf{W} / \mathbf{m ~ K}]$ & $\boldsymbol{C}_{\boldsymbol{p}}[\mathbf{J} / \mathbf{k g K}]$ \\
\hline 1 & 148 & 712 \\
2 & $27.6 \times 10^{3}(T-10.7)+4.5$ & $(((T+120) /(240+0.8$ \\
& & $(T+120)))+0.01) \times 10^{3}$ \\
3 & $1.3 \times 10^{-9}(T-900)^{3}+1.3 \times 10^{-7}$ & $952+(171 T) / 685$ \\
& $(T-900)^{2}+10^{-4}(T-900)+1$ & \\
& &
\end{tabular}

To investigate the thermal behavior, a threedimensional heat equation was considered.

$\rho C_{p} \frac{d T}{d t}=\alpha I+k\left(\frac{\partial^{2} T}{\partial x^{2}}+\frac{\partial^{2} T}{\partial y^{2}}+\frac{\partial^{2} T}{\partial z^{2}}\right)$,

where $\rho$ is the density of the material $\left(\mathrm{kg} / \mathrm{m}^{3}\right), C_{p}$ is the specific heat capacity of the material $(\mathrm{J} / \mathrm{kgK}), \alpha$ is the absorption coefficient of silicon, $I$ is the intensity $\left(\mathrm{W} / \mathrm{m}^{2}\right)$, and $k$ is the thermal conductivity of the material in $(\mathrm{W} / \mathrm{mK})$. The intensity variation is simulated using Beer Lamberts law, $z_{\text {film }}$ represents the thickness of a-Si film, and $R_{x}$ and $R_{y}$ represent the radius of the laser spot.

$I=I_{0} \exp \left[-\alpha\left(z_{\text {film }}-z\right)-\left(\frac{x}{R_{x}}\right)^{2}+\left(\frac{y}{R_{y}}\right)^{2}\right]$,

where $I_{0}$ is the initial intensity.
The equations were solved by considering the thermal conductivity and the specific heat capacity at three different regions as specified in Table 1. The optical absorption coefficient was assumed to be same for all the three regions. Simulation was done using the FEMLAB software.

When a single pulse of a Nd:YAG laser with a laser fluence of $390 \mathrm{~mJ} / \mathrm{cm}^{2}$ at $355 \mathrm{~nm}$ wavelength is incident on the surface of a-Si film, the temperature was estimated to be approximately $1200{ }^{\circ} \mathrm{C}$. Figures 9(a) and (b) show the temperature plots along the central axis in the region of interface obtained from FEMLAB simulation with 50\% and $90 \%$ overlap of the laser spot, respectively. A steeper temperature gradient from 800 to $1260{ }^{\circ} \mathrm{C}$ in the overlap region 2 than that of with the single pulse irradiation of the a-Si film without overlap is observed [11]. The peak temperature value is also estimated to be higher in the overlap region 2 . With $90 \%$ overlapping of the laser beam spot, the peak temperature is further increased to around $1300{ }^{\circ} \mathrm{C}$ as can be seen in Fig. 9(b). The temperature rise in region 2 with $50 \%$ and $90 \%$ overlap is calculated to be around 400 ${ }^{\circ} \mathrm{C}$ and $900{ }^{\circ} \mathrm{C}$, respectively. Further, in the case of $90 \%$ overlap the temperature increase over the area is gradual as compared to that of $50 \%$ overlap. In both the cases, the increase in temperature results in the formation of molten pool of silicon.
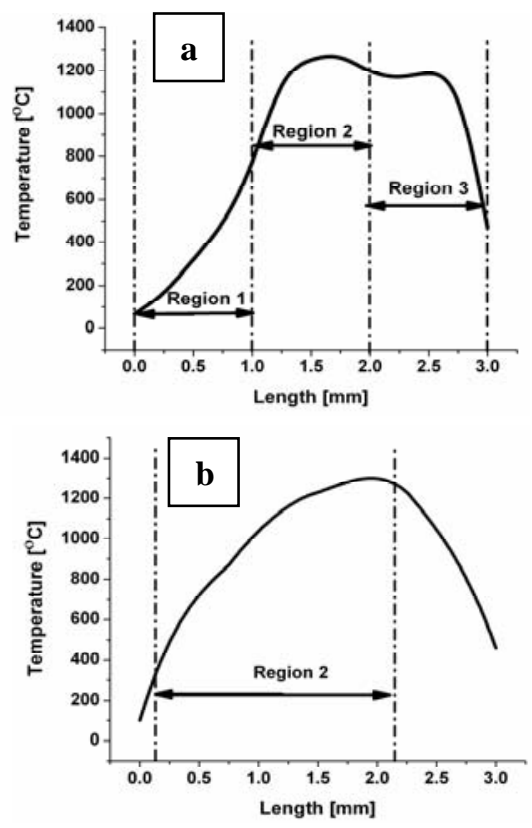

Fig. 9 Surface plot of FEMLAB simulation with a) $50 \%$ overlap b) $90 \%$ overlap.

This strong heating in the interface region (Region 2) is expected to induce a convective motion know as the Marangoni effect as shown in Figs. 10(a) to (d). The Marangoni convection results in a vortex motion and liquid movement towards the region of low temperature as shown in Fig. 10(b). On cooling, due to the decrease in temperature and the surface gradient, the convective motion originating from the Marangoni effect will be damped and the hydrodynamic motion will then evolve as shown in Fig. 10 (c), Capillary waves will be generated due to the surface tension. These capillary waves will exist in the liquefied region until the fused silicon congeals. During cooling the 
amplitude of the surrounding surface will first decrease and transforming to its mirror image by passing a phase where there is practically a smooth surface.

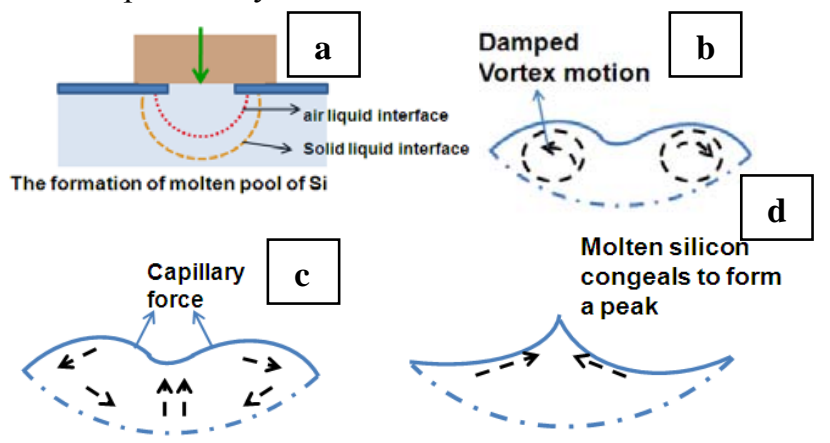

Fig. 10 a) laser annealing of a-Si b) molten Si flow during laser annealing c) molten Si flow during cooling d) growth of crystalline Si peak.

This may result in splashing of material along the liquid trench axis as shown in Fig. 10(d). Hence the formation of peaks depends on the initial temperature of the liquid and the size of the liquid zone. This leads to the formation of a rough pattern at the interface [20-22]. The mechanism was confirmed through the RMS (root mean square of surface roughness) measurement at $90 \%$ and $50 \%$ overlap as shown in Fig. 6. The increase in surface roughness at 90\% overlap is mainly due to the increase in liquid zone at the interface as compared to the $50 \%$ overlap.

\section{Conclusion}

Synthesis of textured polycrystalline-silicon films from amorphous-silicon films is the key issue considered in this paper. In the case of laser annealing and subsequent laser nano-texturing, the first laser pulse is made to incident on the surface of a-Si. After the phase transformation is completed the second laser pulse is made to overlap with the previous irradiated spot to a predetermined extent. The effect of the amount of overlap of $50 \%$ and $90 \%$ of the laser spot diameter is studied. Experimental investigation were carried at three different wavelengths $(355,532$ and $1064 \mathrm{~nm}$ ) of the solid-state $\mathrm{Nd}^{3+}$ :YAG laser. Application of the third harmonics $355 \mathrm{~nm}$ with $90 \%$ overlap on a-Si film resulted in a Raman shift of $522.3 \mathrm{~cm}^{-1}$ and an electrical resistance of $7 \mathrm{k} \Omega$. This is comparable with a-Si films treated with excimer lasers. With $90 \%$ overlap, large number of roughness-peaks is observed and the RMS value is also considerably higher as compared to that with $50 \%$ overlap. On investigating the optical properties, the samples treated with $90 \%$ overlap show a higher absorbance and an improvement in photoconductivity as compared to that of 50\% overlap. Theoretical modeling also shows that, in the case of $50 \%$ overlap a temperature gradient exist along the interface region. On the other hand, by increasing the overlap to $90 \%$ the temperature profile is uniform throughout the interface region resulting in an efficient crystallization for a wider area and leading to the growth of micro-crystals on the surface. This work has shown that the polycrystalline silicon produced with the Nd:YAG laser assisted annealing and nano-texturing 90\% overlap of consecutive laser spot is suitable for photovoltaic applications.

\section{Acknowledgement:}

Authors are grateful to the Kyushu University for the Kyushu University Friendship Scholarship. Authors also thank Dr. D. Nakamura, and Mr. M. Higashihata and Dr. K. Koga for their help during experiments and photoconductivity measurements.

\section{References:}

[1] R.P. Nair, M. Zou: Surface and Coatings Technology, 203 (2008) 675.

[2] L. Carnel, I. Gordon, D. Van Gestel, G. Beaucarne, J. Poortman: Thin Solid Films, 516 (2008) 6839.

[3] L.A. Dobrazan, A. Drygalaa, K. Golombeka, P. Panek, E. Bielan, P. Zie: Journal of Materials Processing Technology, 201 (2008) 291.

[4] H.Y. Chung, C.H. Chen, H.S. Chu: International Journal of Photoenergy, (2008) 5; doi: $10.1155 / 2008 / 540971$.

[5] D. Klinger, E. Lusakowska, D. Zymierska: Material Science in Semiconductor Processing, 9 (2006) 323.

[6] M. Shim, M. Gowtham, M. Kim, D. Seo, J. Yi: Material Science in Semiconductor Processing, 7 (2004) 423.

[7] J.R. Kohler, R. Dassow, R.B. Bergmann, J. Krinke, H.P. Strunk, J.H. Werner : Thin Solid Films, 337 (1999) 129.

[8] I. Tsunoda , R. Matsuura , M. Tanaka, H. Watanabe, T. Sameshima, M. Miyao: Thin Solid Films, 508 (2006) 9.

[9] A. Mittiga, L. Fornarini, R. Carluccio: Applied Surface Science, 154-155 (2000) 112.

[10] I.A. Palani, N.J. Vasa, M. Singaperumal: Material Science in Semiconductor Processing, 11 (2008) 107.

[11] I.A. Palani, N.J. Vasa, M. Singaperumal, T. Okada: Thin Solid Films, 15(2010)518.

[12] T. Chiba, R. Koomura, A. Mori: Japanese Journal of Applied Physics, 39 (2000) 4803.

[13] G. Aichmayr D. Toet, M. Mulato, P.V. Santos, A. Spangenberg, S. Christiansen, M. Albrecht, H.P. Trunk: Journal of Applied Physics, 85 (1999) 19.

[14] Y.F. Lu, Z.F. He, D. Liu: Proc. SPIE Laser Processing of Materials and Industrial Applications, China, 245 (1998) 3550.

[15] J. Svensson, N.M. Bulgakova, O.A. Nerushev, E.E.B. Campbell: Physical Review B, 7, (2006), 205413.

[16] Y.P. Meshcheryakov, N.M. Bulgakova: Applied Physics A, 363 (2006), 82.

[17] N. Biancoa, O. Manca: International Journal of Thermal Sciences, 43 (2004) 611.

[18] J.M. Cole, P. Humphreys, L.G. Earwaker: Vacuum 34 (1984) 871.

[19] E. Gatskevich, P. Rikryl, G. Ivlev : Mathematics and Computers in Simulation, 76 (2007) 65 .

[20] J.C. Conde, P. Gonzalez, F. Lusquin, S. Chiussi, J. Serra : Applied Surface Science 248 (2005) 455.

[21] B.S. Yilbas: Physica A 293 (2001) 157.

[22] A. Fabijańska, F. Kostrubiec, T. Koszmider, R. Pawlak, M.Tomczyk, M.Walczak: Proc. of International Conference Microtechnology and Thermal Problems in Electronics, (MicroTherm'2007), Polska, 167.

(Received: July 10, 2009, Accepted: May 11, 2010) 